\title{
Associação genética da prenhez aos 16 meses com o peso à desmama e o ganho de peso em animais da raça Nelore
}

\author{
Luciana Shiotsuki ${ }^{1}$, Josineudson Augusto II de Vasconcelos Silva ${ }^{2}$, Lucia Galvão de \\ Albuquerque $3,4,5$
}

\author{
1 Doutoranda em Genética e Melhoramento Animal, FCAVIUNESP - Jaboticabal, SP. Bolsista FAPESP. \\ 2 Alta Genetics Brasil, Uberaba, MG. \\ ${ }^{3}$ Faculdade de Ciências Agrárias e Veterinárias, FCAV/UNESP - Jaboticabal, SP. \\ ${ }^{4}$ Pesquisadora CNPq. \\ 5 Pesquisadora do INCT - CA.
}

RESUMO - Objetivou-se verificar a possibilidade de utilização da prenhez de novilhas aos 16 meses (Pr16) como critério de seleção e as possíveis associações genéticas entre prenhez em novilhas aos 16 meses e o peso à desmama (PD) e o ganho de peso médio da desmama ao sobreano (GP). Foram realizadas análises uni e bicaracterísticas para estimação dos componentes de co-variância, empregando-se um modelo animal linear para peso à desmama e ganho de peso da desmama ao sobreano e não-linear para $\operatorname{Pr} 16$. A estimação dos componentes de variância e da predição dos valores genéticos dos animais foi realizada por Inferência Bayesiana. Distribuições "flat" foram utilizadas para todos os componentes de covariância. As estimativas de herdabilidade direta para Pr16, PD e GP foram 0,50; 0,24 e 0,15, respectivamente, e a estimativa de herdabilidade materna para o PD, de 0,07. As correlações genéticas foram -0,25 e 0,09 entre Pr16, PD e GP, respectivamente, e a correlação genética entre Pr16 e o efeito genético materno do PD, de 0,29 . A herdabilidade da prenhez aos 16 meses indica que essa característica pode ser utilizada como critério de seleção. As correlações genéticas estimadas indicam que a seleção por animais mais pesados à desmama, a longo prazo, pode diminuir a ocorrência de prenhez aos 16 meses de idade. Além disso, a seleção para maior habilidade materna favorece a seleção de animais mais precoces. No entanto, a seleção para ganho de peso da desmama ao sobreano não leva a mudanças genéticas na precocidade sexual em fêmeas.

Palavras-chave: bovino de corte, inferência bayesiana, precocidade sexual

\section{Genetic associations of heifer pregnancy at 16 months with weaning weight and average daily gain from weaning to yearling in Nellore cattle}

\begin{abstract}
The objective of the present study was to determine the possible use of heifer pregnancy at 16 months (HP16) as a selection criterion and its possible genetic associations with weaning weight (WW) and average daily gain from weaning to yearling (ADGWY). Covariance components were estimated by uni and bivariate animal models assuming a linear model for weaning weight and average daily gain from weaning to yearling and a nonlinear for HP16. Variance components and breeding values were estimated using Bayesian inference. Flat distributions were used for all (co)variance components and genetic correlations. The estimates of heritability direct for HP16, WW and ADGWY were 0.50; 0.24 and 0.15; respectively. And the maternal heritability estimate for the WW was 0.07; The genetic correlations were -0.25 and 0.09 ; between HP16, WW and ADGWY, respectively; and the genetic correlation between HP16 and maternal effects of WW was 0.29. The heritability estimate of heifer pregnancy at 16 months indicated that the trait can be used as a selection criterion. The genetic correlation estimates indicated that the selection for heavier animals at weaning, in the long-term, could decrease the occurrence of heifer pregnancy at 16 months. Moreover, the selection for animals with greater maternal ability can favor the selection for precocious animals. However, the selection for average daily gain from weaning to yearling will not cause genetic change in female sexual precocity.
\end{abstract}

Key Words: bayesian inferency, beef cattle, sexual precocity

\section{Introdução}

O peso em diferentes idades é uma característica de crescimento utilizada há muitos anos como critério de seleção. O peso à desmama é extremamente importante, pois tem correlação com o peso final (Ferraz Filho et al., 2002; Malhado et al., 2002; Santos et al., 2005). No entanto, esta característica não é influenciada somente 
pelo de sempenho do animal, mas também pelo ambiente materno, representado pela produção de leite e pela habilidade materna (Meyer, 1992).

Quanto mais rápida a taxa de crescimento dos animais, mais curto o ciclo de produção e maior a possibilidade de reduzir os custos de manutenção na propriedade (Marcondes et al., 2000). Desse modo, o cálculo do ganho de peso médio auxilia na seleção dos animais, pois possibilita a escolha de animais mais precoces (Marcondes et al., 2000; Garnero et al., 2001).

A seleção de animais com base nas características de desenvolvimento ponderal é utilizada há muitos anos e estas características foram facilmente incluídas como critério de seleção. No entanto, a implantação de características reprodutivas tem sido mais lenta (Pereira et al., 2001), em virtude das baixas estimativas de herdabilidade de algumas, da impossibilidade de mensuração em ambos os sexos e, no caso de características selecionadas por meio das fêmeas, verifica-se grande intervalo de gerações para se obter resposta à seleção.

A prenhez de novilhas é uma característica binária, com valor 1 (sucesso) para as novilhas que conceberam e pariram e 0 (fracasso) para as novilhas que falharam depois de expostas ao touro na estação de monta. Desta forma, é possível obter medida de todas as contemporâneas, ou seja, todas as fêmeas são incluídas nas análises. Além disso, estimativas de herdabilidade de moderadas a altas têm sido encontradas na literatura (Silva et al., 2003; Silva et al., 2005).

Estimativas acuradas de variâncias e co-variâncias das características de precocidade sexual são fundamentais para permitir sua inclusão em índices de seleção. Portanto, o objetivo com este trabalho foi verificar a possibilidade de utilização da taxa de prenhez de novilhas aos 16 meses (Pr16) como critério de seleção e suas possíveis associações genéticas com peso à desmama e ganho de peso da desmama ao sobreano, visando fornecer subsídios necessários ao desenvolvimento de índices de seleção econômicos incluindo estas características.

\section{Material e Métodos}

Os registros utilizados são de animais da raça Nelore nascidos no período de 1984 a 2004, pertencentes ao arquivo zootécnico da Agropecuária Jacarezinho Ltda. Os objetivos principais na Agropecuária são a venda de reprodutores jovens e de animais para abate.

O manejo alimentar para touros e vacas é somente a pasto, com sal mineral à vontade. A estação de monta normal tem início em torno da segunda quinzena de novembro e, para as vacas, tem duração de aproximadamente 70 dias, enquanto, para as novilhas, tem duração de aproximadamente 60 dias, com inseminação artificial e reprodutor múltiplo. A partir de 1990, foi implantada uma estação de monta antecipada para as novilhas entre os meses de fevereiro e abril (60 dias), desse modo, as novilhas são expostas em média aos 16 meses de idade, em regime de reprodutor múltiplo, com relação touro:vaca de 1:30. Todas as novilhas são expostas à reprodução,independentemente do peso e da condição corporal. Quando não concebem na estação de monta antecipada, as novilhas são expostas novamente na estação de monta normal e, caso não emprenhem, são descartadas.

As novilhas são avaliadas quanto à prenhez, por palpação retal, aproximadamente 60 dias após o término da estação de monta antecipada. Os critérios de descarte de fêmeas no rebanho são: falha reprodutiva da novilha na estação de monta antecipada e normal, consecutivamente; falha da vaca em um ano, baixa avaliação de desempenho de progênies e pequena porcentagem por sanidade.

A característica prenhez aos 16 meses (Pr16) foi obtida para todas as fêmeas com registro de peso à desmama no arquivo de análise, considerando que todas as fêmeas pesadas à desmama tiveram condições de emprenhar. Conforme o manejo da fazenda, todas as fêmeas que permaneceram até o sobreano foram expostas ao acasalamento. Assim, a prenhez aos 16 meses foi definida com base na concepção e parição da novilha, desde que tenha entrado na estação de monta aos 16 meses de idade. É uma característica binária; ou seja, às novilhas que pariram com menos de 31 meses foi atribuído o valor 1 (sucesso) e àquelas que falharam, valor 0 (fracasso).

As características peso à desmama (PD) e ganho de peso da desmama ao sobreano (GP) foram mensuradas em ambos os sexos e o ganho de peso da desmama até ao sobreano (GP) foi obtido pela fórmula:

$$
\mathrm{GP}=\frac{\mathrm{PS}-\mathrm{PD}}{\mathrm{Ips}-\mathrm{Ipd}},
$$

em que PS é peso ao sobreano; PD, peso à desmama; Ips, idade do peso ao sobreano; Ipd, idade do peso à desmama.

$\mathrm{Na}$ consistência dos dados, foram descartadas as informações de grupos contemporâneos (GC) com menos de quatro animais para as características de peso à desmama e ganho de peso e medidas superiores ou inferiores à média do grupo de contemporâneos, mais ou menos 3,5 desvios-padrão, respectivamente. Todos os animais tinham pelo menos a mãe conhecida. Para a característica prenhezaos 16 meses, observou-se que, dentro dos grupos de contemporâneos, havia variabilidade, ou seja, não 
apresentavam todos os animais com somente valores 0 (fracasso) ou 1 (sucesso). No arquivo trabalhado, entretanto, não houve nenhum caso de grupo de contemporâneos sem variabilidade.

O número de animais que possuíam ambas as características foi de 28.084 para prenhez aos 16 meses e peso à desmama; e 23.519 para as medidas de prenhez aos 16 meses e ganho de peso da desmama ao sobreano (Tabela 1).

Foram utilizadas análises uni e bicaracterísticas para estimar componentes de co-variância, empregando-se modelo de limiar para prenhez de novilhas e modelo animal linear para peso à desmama e ganho de peso. Para a estimação dos componentes de variâncias e predição dos valores genéticos dos animais, por Inferência Bayesiana, utilizou-se o software MTGSAM (Multiple Trait Gibbs Sampling in Animal Models), desenvolvido por Van Tassell \& Van Vleck (1998).

O modelo utilizado é descrito da seguinte forma:

$$
\mathrm{y}=\mathrm{X} \beta+\mathrm{Z}_{1} \mathrm{a}+\mathrm{Z}_{2} \mathrm{~m}+\mathrm{e}
$$

em que: $y=$ vetor de observações (prenhez de novilhas, peso à desmama e ganho de peso); $\beta=$ vetor de efeitos fixos; $\mathrm{a}=$ vetor dos efeito genético aditivo aleatório do animal; $X=$ matriz de incidência dos efeitos fixos, que associa o vetor $\beta$ ao vetor $y ; Z_{1}=$ matriz de incidência dos efeitos aleatórios genéticos diretos (prenhez de novilhas, peso à desmama e ganho de peso); $Z_{2}=$ matrizdeincidência dos efeitos aleatórios genéticos maternos (peso à desmama); $\mathrm{m}=$ vetor dos efeitos aleatórios genéticos maternos (peso à desmama); e = vetor de resíduo;

Assumiu-se que os efeitos genéticos diretos e residuais não foram correlacionados entre si. Para as características com distribuição contínua de peso à desmama e ganho de peso, assumiu-se que:

$\mathrm{y} \sim \mathrm{N}\left(\mathrm{X} \beta+\mathrm{Za}, \mathrm{I} \sigma_{\mathrm{e}}^{2}\right) ;$

$\mathrm{a} \mid \sigma_{\mathrm{a}}^{2} \sim \mathrm{N}(0, \mathrm{~A} \otimes \mathrm{G}) \mathrm{e}$

$\mathrm{e} \mid \sigma_{\mathrm{e}}^{2} \sim \mathrm{N}\left(0, \mathrm{I} \sigma_{\mathrm{e}}^{2}\right)$ em que: $\sigma_{\mathrm{a}}^{2}=$ variância genética aditiva direta; $\mathrm{A}=$ matriz dos coeficientes de parentesco entre os animais; $\sigma_{\mathrm{e}}^{2}=$ variância residual; $\mathrm{I}=$ matriz identidade; $\mathrm{G}=$ matriz de covariâncias genéticas.

A prenhez de novilhas é uma característica categórica ou de limiar que, segundo Falconer \& Mackay (1996), possui distribuição contínua subjacente, com um limiar, o qual torna a expressão da característica descontínua. Assim, neste caso, empregou-se um modelo de limiar assumindo-se que a distribuição subjacente (U) é determinada por:

$\mathrm{U} \sim \mathrm{N}\left(\mathrm{X} \beta+\mathrm{Za}, \mathrm{I} \sigma_{\mathrm{e}}^{2}\right)$

As distribuições iniciais para os efeitos genéticos e os residuais seguem distribuições normais multivariadas:

$\mathrm{p}\left(\mathrm{a} \mid \sigma_{\mathrm{a}}^{2}\right) \sim \mathrm{N}\left(0, \sigma_{\mathrm{a}}^{2}\right)$

$\mathrm{p}\left(\mathrm{a} \mid \sigma_{\mathrm{e}}^{2}\right) \sim \mathrm{N}\left(0, \mathrm{I} \sigma_{\mathrm{e}}^{2}\right)$

Como $\sigma_{\mathrm{e}}^{2}$ não é estimável (Gianola \& Foulley, 1983), atribuiu-se a essa característica o valor arbitrário 1,0 e definiram-se distribuições a priori uniformes para os efeitos fixos ( $\left.b^{\prime}=E F^{\prime}\right)$ e para $\sigma_{a}^{2}$.

De acordo com Gianola \& Foulley (1983) e Harville \& Mee (1985), após a definição dos parâmetros do modelo, o encadeamento entre as duas escalas (categórica e contínua) pode ser estabelecido de forma que a probabilidade de uma observação estar na primeira categoria é proporcionala:

$$
\mathrm{P}\left(\mathrm{y}_{\mathrm{r}}=0 \mid \mathrm{t}, \theta\right)=\mathrm{P}\left(\mathrm{U}_{\mathrm{r}}<\mathrm{t} \mid \mathrm{t}, \theta\right)=\phi\left(\left(\mathrm{t}-\mathrm{W}_{\mathrm{r}}^{\prime} \theta\right) / \sigma_{\mathrm{e}}\right),
$$

em que: $y_{\mathrm{r}}=$ variável-resposta para a r-ésima observação, tomando valores 0 ou 1 se a observação pertence à primeira ou à segunda categoria, respectivamente; $\mathrm{t}=$ valor do limiar que, por não ser estimável, é fixado com valor arbitrário; $\mathrm{U}_{\mathrm{r}}=$ valor da variável subjacente para a mencionada observação; $\phi()$ = função de distribuição cumulativa de

\begin{tabular}{|c|c|c|c|}
\hline Característica & Prenhez aos 16 meses & Peso à desmama & Ganho de peso da desmama ao sobreano \\
\hline Número de observações & 28.084 & 111.521 & 48.056 \\
\hline Média & $13,33^{\mathrm{a}}$ & 171,24 & 0,31 \\
\hline Idade mínima e máxima & 410: 632 & 150: 270 & $275: 424$ \\
\hline № de grupos contemporâneos & 15 & 2.134 & 1.060 \\
\hline № de pais & 276 & 412 & 358 \\
\hline № de mães & 16.783 & 39.908 & 22.660 \\
\hline № de animais com & 16.032 & 72.251 & 18.649 \\
\hline \multicolumn{4}{|l|}{ paternidade desconhecida } \\
\hline № de animais na matriz de parentesco & 128.933 & 128.933 & 67.068 \\
\hline
\end{tabular}

Tabela 1 - Descrição do arquivo de dados para as características avaliadas

${ }^{a}$ medida em porcentagem $(\%)$ 
uma variável normal padrão; $\mathrm{W}_{\mathrm{r}}^{\prime}=$ vetor coluna de incidência que une $\theta$ à r-ésima observação; $\boldsymbol{\theta}=\left(\mathrm{b}^{\prime}, \mathrm{a}^{\prime}\right)$, vetor dos parâmetros de locação de ordem s com b (definidos sob ponto de vista frequentista, como efeitos fixos) e a (como efeito aleatório).

No modelo para prenhez aos 16 meses, incluíram-se os efeitos sistemáticos de grupo contemporâneo (GC: fazenda + ano de nascimento); o efeito linear da idade na entrada da estação de monta como co-variável; além do efeito animal e do resíduo.

$\mathrm{Na}$ avaliação da característica peso à desmama, foram considerados os efeitos de grupo de contemporâneos (fazenda + ano e mês de nascimento + sexo + grupo de manejo na desmama); a co-variável linear e quadrática da idade no momento da mensuração e idade da mãe ao parto (dias), além do efeito animal e resíduo. Na análise do ganho de peso da desmama ao sobreano, foram considerados os efeitos de grupo de contemporâneos (fazenda + ano e mês de nascimento + sexo + grupo de manejo na desmama e ao sobreano); a co-variável linear da idade no momento da mensuração, além do efeito animal e resíduo.

Nas análises uni e bicaracterísticas entre prenhez aos 16 meses e peso ao sobreano e ganho de peso, foram
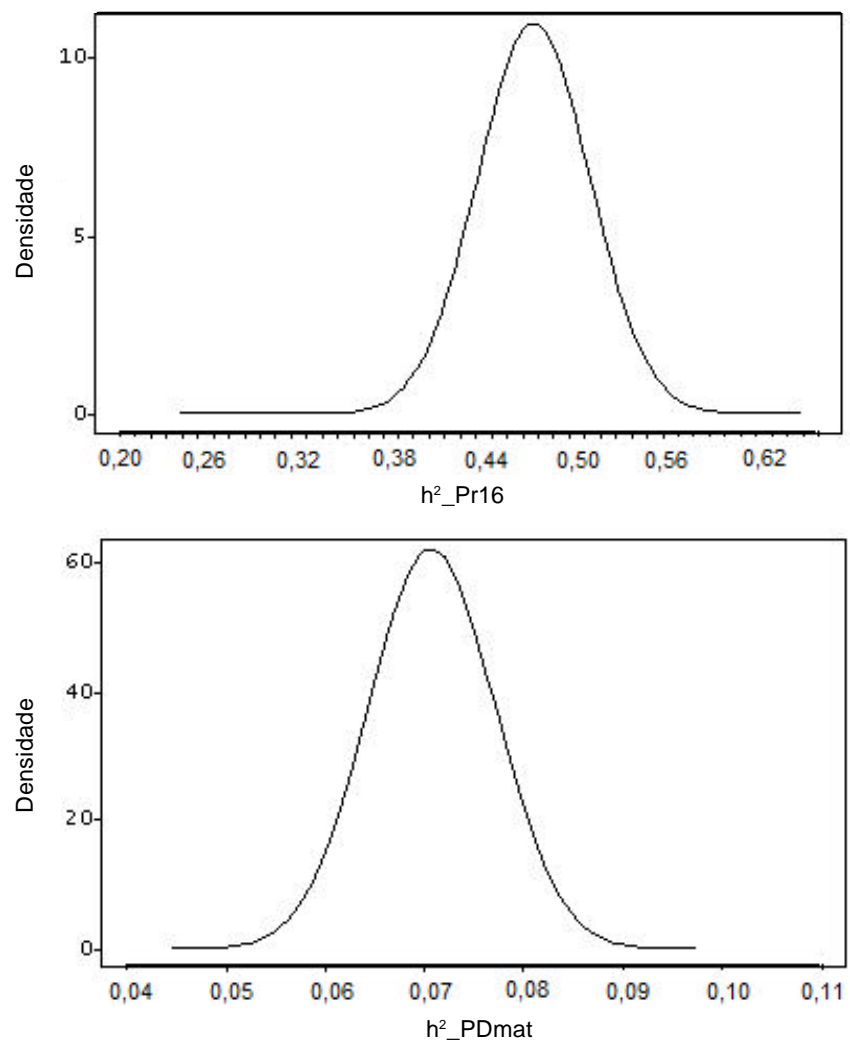

computadas duas cadeias independentes de 300.000 ciclos. Foram retirados os 5.000 primeiros ciclos como período de descarte amostral para análises das distribuições a posteriori das herdabilidades. Distribuições uniformes foram utilizadas para todos os componentes de co-variâncias e correlações genéticas. Foram assumidas herdabilidade de 0,20 e correlação genética de 0,30 como valores iniciais para e entre as características. O critério de convergência foi de $10^{-12}$.

As estimativas da distribuição a posteriori foram analisadas quanto a sua convergência pelo programa Gibbanal (Van Kaam, 1998), que utiliza o teste Raftery \& Lewis (1992), baseado na baixa correlação serial entre os ciclos para indicar a convergência da cadeia.

\section{Resultados e Discussão}

$\mathrm{Na}$ análise da densidade das estimativas a posteriori da fase estacionária pelo programa Gibbanal (Van Kaam, 1998), os valores das medidas de tendência central com base nos ciclos considerados pelo programa do Gibbanal foram idênticos aos obtidos utilizando-se a cadeia completa (Figuras 1 e 2).
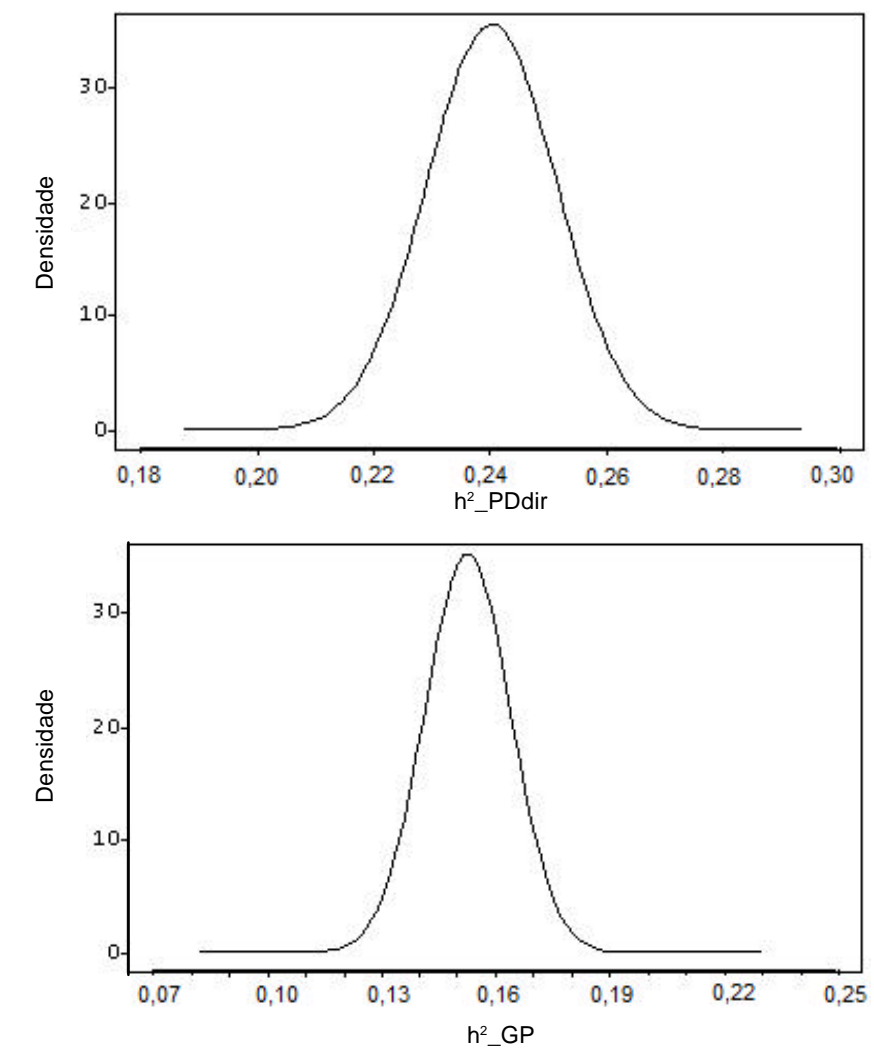

Figura 1 - Densidade a posteriori das herdabilidades para efeito genético direto da prenhez aos 16 meses (Pr16), peso à desmama (PDdir) e ganho peso (GP), e para efeito genético materno do peso à desmama (PDmat). 

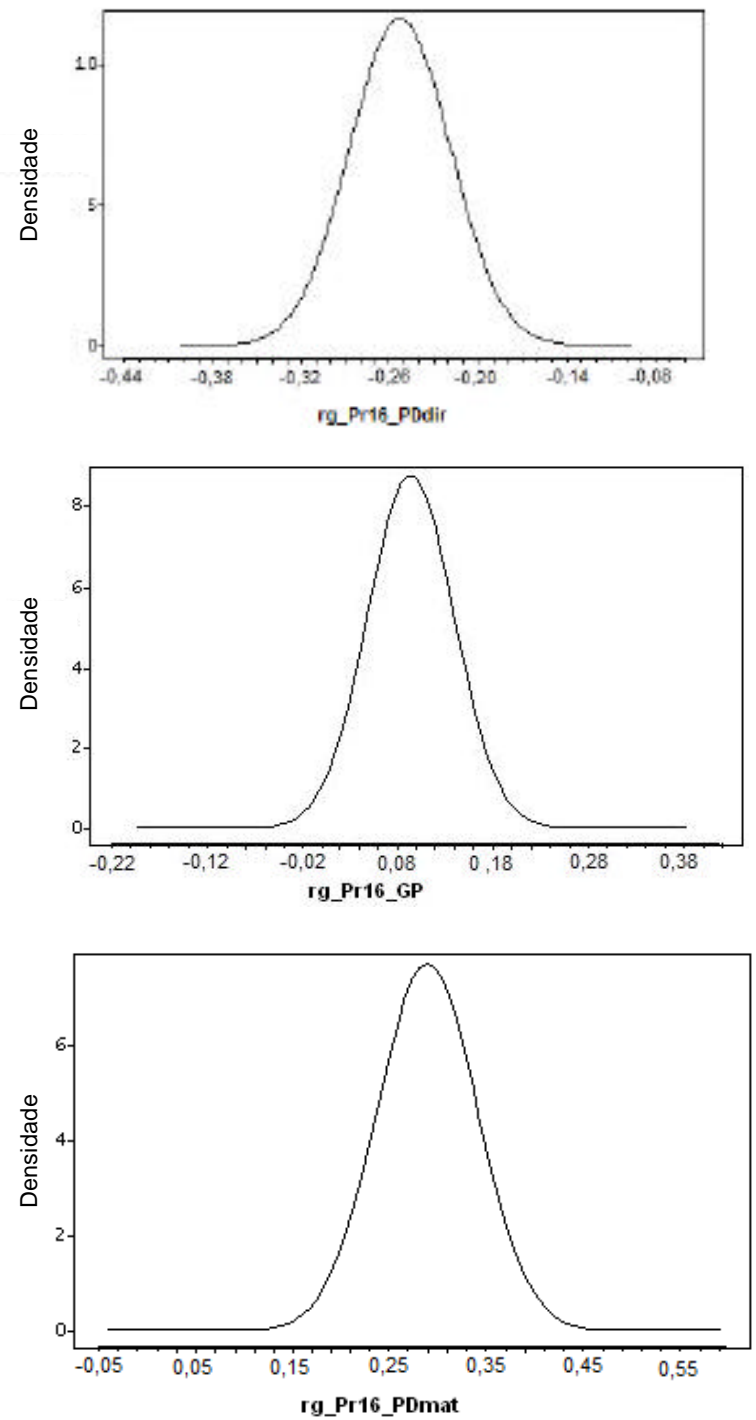

Figura 2 - Densidade a posteriori da correlação genética entre os efeitos direto da prenhez aos 16 meses (Pr16), pe so à desmama (PDdir) e ganho peso (GP), e entre efeito direto da prenhez aos 16 meses (Pr16) e o efeito materno do peso à desmama (PDmat).

As estimativas de herdabilidade foram semelhantes nas análises uni (Tabela 2) e bicaracterísticas (Tabela 3). Os limites para os intervalos de maior densidade a posteriori (em inglês, 'Higher Posterior Density') contendo $90 \%$ das observações foram pequenos, principalmente para as estimativas de herdabilidade (Tabelas 2 e 3 ).

Os valores das estimativas médias a posteriori da herdabilidade para prenhez aos 16 meses (Tabelas 2 e 3) foram próximos aos descritos na literatura para animais zebuínos (Atencio, 2000; Eler et al., 2002; Silva \& Albuquerque, 2004) e indicam que a característica prenhez aos 16 meses responderá eficientemente à seleção.
Tabela 2 - Estimativas a posterioridas herdabilidades para efeito direto da prenhez de novilhas aos 16 anos (Pr16), peso à desmama (PDdir), e ganho de peso da desmama ao sobreano (GP), e para efeito materno do peso à desmama (PDmat) de animais Nelore em análises unicaracterísticas

Parâmetro Média \pm Desvio-padrão Moda Mediana RMD_90\%

\begin{tabular}{lllll}
\hline $\mathrm{h}^{2} \_$Pr16 & $0,50 \pm 0,03$ & 0,49 & 0,50 & 0,43 a 0,56
\end{tabular}

$\mathrm{h}^{2}{ }_{-}$PDdir $\quad 0,24 \pm 0,01 \quad 0,23 \quad 0,24 \quad 0,21$ a 0,26

$\mathrm{h}^{2}$ _PDmat $\quad 0,07 \pm 0,01 \quad 0,07 \quad 0,07 \quad 0,05$ a 0,08

$\mathrm{h}^{2}{ }_{-} \mathrm{GP} \quad 0,15 \pm 0,01 \quad 0,15 \quad 0,15 \quad 0,13$ a 0,17

$\mathrm{h}^{2}{ }_{-}=$herdabilidade de; $\mathrm{DP}=$ desvio-padrão; $\mathrm{RMD} \_90 \%$ = região de maior densidade de probabilidade contendo $90 \%$ das observações.

Tabela 3 - Estimativas a posteriori dos parâmetros genéticos para efeito direto da prenhez de novilhas aos 16 meses (Pr16), peso à desmama (PDdir) e ganho de peso da desmama ao sobreano (GP), e para efeito materno do peso à desmama (PDmat) de animais Nelore em análises bicaracterísticas

Parâmetro Média \pm Desvio-padrão Moda Mediana RMD $90 \%$

\begin{tabular}{lllll}
\hline $\mathrm{h}^{2} \_$Pr16* & $0,51 \pm 0,03$ & 0,52 & 0,51 & 0,46 a 0,56
\end{tabular}

$\begin{array}{llllll}\mathrm{h}^{2} \text { PDdir } & 0,24 \pm 0,01 & 0,24 & 0,24 & 0,22 \text { a } 0,26\end{array}$

$\mathrm{h}^{2}{ }_{2}$ PDmat $\quad 0,07 \pm 0,01 \quad 0,07 \quad 0,07 \quad 0,06$ a 0,08

$\begin{array}{lllll}\mathrm{h}^{2} \text { GP } & 0,15 \pm 0,01 & 0,16 & 0,15 & 0,13 \text { a } 0,17\end{array}$

rg_Pr16_PDdir $\quad-0,25 \pm 0,03 \quad-0,24 \quad-0,25 \quad-0,32$ a $-0,19$

rg_Pr16_PDmat $0,29 \pm 0,05 \quad 0,29 \quad 0,29 \quad 0,18$ a 0,38

$\begin{array}{lllll}\text { rg_Pr16_GP } & 0,09 \pm 0,05 & 0,09 & 0,10 & 0,02 \text { a } 0,17\end{array}$

re_Pr16_PDdir $\quad 0,24 \pm 0,00 \quad 0,24 \quad 0,24 \quad 0,24$ a 0,25

re_Pr16_Pdmat $\quad 0,04 \pm 0,00 \quad 0,04 \quad 0,04 \quad 0,03$ a 0,04

re_Pr16_GP $\quad 0,30 \pm 0,01 \quad 0,30 \quad 0,30 \quad 0,28$ a 0,31

$\mathrm{h}^{2}{ }_{-}=$herdabilidade; $\mathrm{DP}=$ desvio-padrão; RMD_90\%= Região de maior densidade de probabilidade contendo $90 \%$ das observações; * = valores obtidos da análise bicaracterística com PD; $\mathrm{rg}_{-}=$correlação genética; re - $_{\text {- }}$ correlação residual

Segundo Silva et al. (2005), esta característica é indicada para seleção de touros quando o intuito é aumentar a precocidade sexual de fêmeas. Entretanto, como a prenhez de novilhas aos 16 meses é mensurada apenas nas fêmeas, a avaliação genética dos touros deve ser realizada com base no desempenho de suas filhas, o que pode retardar a resposta à seleção.

As estimativas de herdabilidade obtidas para ganho de peso da desmama ao sobreano e peso à desmama, tanto para o efeito direto como o materno, estão dentro da amplitude de valores descrita para animais de raças zebuínas (Eler et al., 1995, Marcondes et al., 2000, Albuquerque \& Meyer, 2001; Gunsky et al., 2001; Pereira et al., 2001; Ferraz Filho et al., 2002; Marcondes et al., 2002; Panetto et al., 2002; Kock et al., 2004; Knackfuss et al., 2006; Quintero et al., 2007).

A estimativa a posteriori da correlação genética entre os efeitos genéticos diretos da prenhez aos 16 meses e o peso à desmama foi moderada e negativa (Tabela 3), o que está de acordo com o descrito por Pereira et al. (2001), que estimaram correlação genética positiva $(0,10)$ entre 
peso à desmama e idade ao primeiro parto de fêmeas expostas ao touro pela primeira vez em torno de 14 meses de idade. Essa correlação sugere que, a longo prazo, a seleção para animais mais pesados à desmama levará à diminuição da taxa de prenhez aos 16 meses. Assim, a seleção para prenhez aos 16 meses pode trazer benefícios para antecipação do início da vida reprodutiva das fêmeas, mas também poderá, a longo prazo, resultar em animais mais leves à desmama. Os limites biológicos e técnicos de se diminuir a idade à puberdade nos sistemas de produção ainda precisam ser explorados.

Os valores obtidos para a correlação genética entre o efeito genético direto da prenhez aos 16 meses e o efeito genético materno do peso à desmama (PDmat) foram moderados e positivos (Tabela 3 ). Essa correlação sugere que a seleção para prenhez aos 16 meses, além de trazer benefícios para antecipação do início da vida reprodutiva das fêmeas, produzirá animais com maior habilidade materna. Os resultados deste trabalho confirmam os encontrados por Everling et al. (2001), que encontraram correlação genética entre efeito materno para peso à desmama e perímetro escrotal moderado e positivo $(0,21)$.

A correlação genética entre prenhez aos 16 meses e ganho de peso foi positiva, mas baixa (Tabela 3 ). Desta forma, a utilização do ganho de peso como critério de seleção, a curto ou médio prazo, não deve ocasionar mudanças genéticas importantes na precocidade sexual. Os dados obtidos neste trabalho confirmam os encontrados por Peña et al. (2001), que verificaram correlações genéticas positivas e baixas entre ganho de peso pós-desmama e perímetro escrotal $(0,33)$. Pereira et al. (2001) também verificaram correlações genéticas baixas entre ganho de peso da desmama ao sobreano com idade ao primeiro parto (aos 14 e 16 meses) e dias para o parto $(-0,08 ;-0,03 ;-0,11$; respectivamente). Esses autores concluíram que, se as fêmeas atingirem valor mínimo de ganho de peso, não haveria danos para o início da puberdade, assim, todos os animais teriam desempenho semelhante.

As estimativas médias de correlações residuais entre efeito genético direto, tanto para prenhez aos 16 meses e peso à desmama, quanto para prenhez aos 16 meses e ganho de peso, resultaram em valores positivos e moderados. A estimativa de correlação residual entre prenhez aos 16 meses e o efeito materno do peso à desmama, no entanto, foi baixa (Tabela 3). Estimativas de correlação residual incluem as associações decorrentes dos efeitos de ambiente, não considerados no modelo de análise, e os efeitos genéticos não-aditivos.
Os resultados indicam que a prenhez aos 16 meses pode ser incluída em programas de melhoramento animal por apresentar valores estimados de herdabilidade de magnitude alta, o que indica a possibilidade de obtenção de ganhos genéticos para a precocidade sexual em fêmeas. Os valores obtidos para as correlações genéticas entre prenhez aos 16 meses e peso à desmama, e prenhez aos 16 meses e ganho de peso, indicaram que, quando o objetivo for promover mudanças genéticas, tanto para reprodução como para crescimento, as características prenhez aos 16 meses, peso à desmama e ganho de peso podem ser utilizadas em um índice de seleção, dependendo dos objetivos em cada propriedade.

\section{Conclusões}

A estimativa de herdabilidade da prenhez aos 16 meses indica que essa característica pode ser utilizada como critério de seleção, com possibilidade de promover ganho genético na precocidade sexual das novilhas. A seleção para animais mais pesados à desmama, a longo prazo, pode diminuir a ocorrência de prenhez aos 16 meses de idade. Além disso, a seleção para maior habilidade materna resultaria em resposta correlacionada para precocidade sexual de fêmeas. No entanto, a seleção para ganho de peso da desmama ao sobreano não ocasiona mudanças genéticas na precocidade sexual em fêmeas.

\section{Literatura Citada}

ALBUQUERQUE, L.G.; MEYER, K. Estimates of direct and maternal genetic effects for weights from birth to 600 days of age in Nelore cattle. Journal of Animal Breeding and Genetic, v.118, p.83-92, 2001.

ATENCIO, A.M. Predicción genética de la fertilidad em la hembra cebu. In: CONGRESSO INTERNACIONAL DE TRANSFERÊNCIA TECNOLÓGICA AGROPECUÁRIA, 20., 2000, Assunción. Anales... Assunción: Congresso Internacional de Transferência Tecnológica Agropecuária, 2000. p.29-42.

ELER, J.P.; Van VLECK, L.D.; FERRAZ, J.B.S. Estimation of variances due to direct and maternal effects for growth traits of Nelore catlle. Journal of Animal Science, v.73, p.3253$3258,1995$.

ELER, J.P.; SILVA, J.A. II V.; FERRAZ, J.B.S. et al. Genetic evaluation of the probability of pregnancy at 14 months for Nellore heifers. Journal of Animal Science, v.80, p.49514954, 2002.

EVERLING, D.M.; FERREIRA, G.B.B.; RORATO, P.R.N. Estimativas de herdabilidade e correlação genética para características de crescimento na fase de pré-desmama e medidas de perímetro escrotal ao sobreano em bovinos Angus-Nelore. Revista Brasileira de Zootecnia, v.30, p.2002-2008. 2001.

FALCONER, D.S.; MACKAY, F.C. Introduction to quantitative genetics. 4.ed. England: Longmans Green, Harlow, 1996. 464p.

FERRAZ FILHO, P.B.; RAMOS, A.A.; SILVA, L.O.C. et al. Herdabilidade e correlações genéticas, fenotípicas e ambientais 
para pesos em diferentes idades de bovinos da raça Tabapuã Archives of Veterinary Science, v.7, n.1, p.65-69, 2002.

GARNERO, A.D.V.; LÔBO, R.B.; BEZERRA, L.A.F. et al Comparação entre Alguns Critérios de Seleção para Crescimento na Raça Nelore. Revista Brasileira de Reprodução Animal, v.30, n.3, p.714-718, 2001

GIANOLA, D.; FOULLEY, J.L. Sire evaluation for ordered categorical data with a threshold model. Genetics Selection Evolution, v.15, p.201-224, 1983

GUNSKY, R.J.; GARNERO, A.D.V.; BORJAS, A.D.L.R. et al. Estimativas de parâmetros genéticos para características incluídas em critérios de seleção em gado Nelore. Revista Ciência Rural, v.32, n.4, p.603-607, 2001.

HARVILLE, D.A.; MEE, R.W. A mixed model procedure for analyzing ordered categorical data. Journal of Dairy Science, v.68, p.1763-1769, 1985.

HOLANDA, M.C.R.; BARBOSA, S.B.P.; RIBEIRO, A.C. et al. Tendências genéticas para crescimento em bovinos Nelore em Pernambuco, Brasil. Archivos de Zootecnia, v.53, p.185-194. 2004.

KNACKFUSS, F.B.; RAZOOK, A.G.; MERCADANTE, M.E.Z. et al Seleção para peso pós-desmama em um rebanho Gir. 2. Estimativas de variâncias e parâmetros genéticos dos efeitos direto e materno para características de crescimento. Revista Brasileira de Zootecnia, v.35, n.3, p.726-732. 2006.

KOCH , R.M.; CUNDIFF, L.V.; GREGORY, K.E. et al. Genetic response to selection for weaning weight or yearling weight or yearling weight and muscle score in Hereford cattle: efficiency of gain, growth, and carcass characteristics. Journal of Animal Science, v.82, p.668-682, 2004.

MALHADO, C.H.M.; SOUZA, J.C.; SILVA, L.O.C. et al. Correlações genéticas, fenotípicas e de ambiente entre os pesos de várias idades em bovinos da raça Guzerá no estado de São Paulo. Archives of Veterinary Science, v.7, n.1, p.71-75, 2002.

MARCONDES, C.R. Análise de alguns critérios de seleção para características de crescimento na raça Nelore. 1999. 93f. Dissertação de Mestrado (Mestrado em Zootecnia) - Escola de Veterinária, Universidade Federal de Minas Gerais, Belo Horizonte, 1999.

MARCONDES, C.R.; BERGMANN J.A.; ELER J.P. et al. Análise de alguns critérios de seleção para características de crescimento na raça Nelore. Arquivo Brasileiro de Medicina Veterinária e Zootecnia, v. 52, n.1, p.83-89, 2000.

MARCONDES, C.R.; GAVIO, D.; BITTENCOURT, T.C.C. Estudo de modelo alternativo para estimação de componentes de (co)variância e predição de valores genéticos de características de crescimento em bovinos da raça Nelore. Arquivo Brasileiro de Medicina Veterinária e Zootecnia, v.54, n.1, p.93-99, 2002.
MEYER, K. Variance components due to direct and maternal effects for growth traits of Australian beef cattle. Livestock Production Science, v.31, p.179-203, 1992.

PANET O, J.C.C.; LEMOS, D.C.; BEZERRA, L.A.F. et al. Estudo de características quantitativas de crescimento dos 120 aos 550 dias de idade em gado Nelore. Revista Brasileira de Zootecnia, v.31, n.2, p.668-674, 2002.

PEÑA, C.D.O.; QUEIROZ, S.A.; FRIES, L.A. Comparação entre critérios de seleção sexual e a associação destes com características de crescimento em bovinos de corte. Revista Brasileira de Zootecnia, v.30, n.1, p.93-100, 2001.

PEREIRA, E.; ELER, J.P.; FERRAZ, J.B.S. Análise genética de algumas características reprodutivas e suas relações com o desempenho ponderal na raça Nelore. Arquivo Brasileiro de Medicina Veterinária e Zootecnia, v.53, n.6, p.720-727, 2001.

QUINTERO, J.C.; TRIANA, J.G.; QUIJANO, J.H. Influencia de la inclusión del efecto materno en la estimación de parámetros genéticos del peso al destete en un hato ganado de carne. Revista Colombiana de Ciencias Pecuárias, v.20, p.117-123, 2007.

RAFTERY, A.E., LEWIS, S.M. How many iterations in the Gibbs sampler. In:BERNARDO, J.M.; BERGER, J.O.; DAVID, A.P. et al. (Eds.) Bayesian atatistics . Clarendon Press: Oxford, 1992. v.4, p.763-773.

SANTOS, P.F.; MALHADO, C.H.M.; CARNEIRO, P.L.S. et al. Correlação genética, fenotípica e ambiental em características de crescimento de bovinos da raça Nelore variedade mocha. Archives of Veterinary Science, v.10, n.2, p.55-60, 2005.

SILVA, J. A.II V.; VAN MELIS, M.H.; ELER, J.P. et al. Estimação de parâmetros genéticos para probabilidade de prenhez aos 14 meses e altura na garupa em bovinos da raça Nelore. Revista Brasileira de Zootecnia, v.32, n.5, p.1141-1146, 2003.

SILVA, J.A.II V.; ALBUQUERQUE, L.G. Estudo da prenhez aos 18 meses de idade ao primeiro parto em novilhas Nelore. In: SIMPÓSIO DA SOCIEDADE BRASILEIRA DE MELHORAMENTO ANIMAL, 5., 2004, Pirassununga. Anais... Pirassununga, SBMA, [2004] (CD-ROM).

SILVA, J.A. II V.; DIAS L.T.; ALBUQUERQUE L.G. Estudo genético da precocidade sexual de novilhas em um rebanho Nelore. Revista Brasileira de Zootecnia, v.34, n.5, p.1568-1572, 2005.

Van KAAM, J.B.C.H.M. "GIBANAL" - Analyzing program for Markov Chain Monte Carlo Sequences (Version 2.10). Wageningen: Department of Animal Sciences, Agricultural University, 1998. 4p. (Manual)

Van TASSELL, C.P.; Van VLECK, L.D.; GREGORY, K.E. Bayesian analysis of twinning and ovulation rates using a multiple-trait threshold model and Gibbs sampling. Journal of Animal Science, v.76, n.8, p.2048-2061, 1998 